\title{
Which Osteotomy for Osteonecrosis of the Femoral Head and Which Patient for the Osteotomy?
}

\author{
Young-Kyun Lee, MD, Beomseok Lee, MD, Javad Parvizi, MD*, Yong-Chan Ha, $\mathrm{MD}^{\dagger}$, Kyung-Hoi Koo, MD \\ Department of Orthopedic Surgery, Seoul National University Bundang Hospital, Seongnam, Korea, \\ ${ }^{*}$ Rothman Institute, Thomas Jefferson University Hospital, Philadelphia, PA, USA, \\ ${ }^{\dagger}$ Department of Orthopaedic Surgery, Chung-Ang University College of Medicine, Seoul, Korea
}

Transtrochanteric curved varus osteotomy (TCVO) and transtrochanteric rotational osteotomy (TRO) are joint-preserving procedures for osteonecrosis of the femoral head. The purpose of this review is to provide up-to-date guidelines for the osteotomies. One retrospective comparison revealed that TCVO has shorter operation time, less bleeding, lower incidence of osteophyte formation, and lower rate of secondary collapse. To obtain successful results of the osteotomy, the patient should be younger than 40 years and should have a body mass index of less than $24 \mathrm{~kg} / \mathrm{m}^{2}$. The osteotomy should be performed in early stages of femoral head osteonecrosis before marked collapse of the femoral head. The patient should have a medium-size lesion and an enough viable bone to restore the intact articular surface and subchondral bone in the weight-bearing area.

Keywords: Osteotomy, Femur head necrosis

Femoral head osteonecrosis usually affects adults younger than 50 years and frequently leads to collapse and subsequent osteoarthritis of the hip. ${ }^{1,2)}$ It is becoming more prevalent because of increasing use of steroids in the management of organ transplantation and adjuvant therapy for leukemia and other myelogenous diseases. ${ }^{3-5)}$ This disease frequently necessitates total hip arthroplasty (THA). ${ }^{6-8)}$ As alternatives to THA, several osteotomies of the proximal femur have been introduced to preserve the hip joint. ${ }^{9-11)}$ These techniques move the necrotic portion from the weight-bearing region to a non-weight-bearing region. Among them, transtrochanteric curved varus osteotomy $(\mathrm{TCVO})^{11)}$ and transtrochanteric rotational osteotomy $(\mathrm{TRO})^{9)}$ are well-known and popular in use.

Received February 19, 2019; Accepted March 20, 2019

Young-Kyun Lee and Beomseok Lee contributed equally to this work as first authors.

Correspondence to: Kyung-Hoi Koo, MD

Department of Orthopedic Surgery, Seoul National University Bundang Hospital, 82 Gumi-ro 173beon-gil, Bundang-gu, Seongnam 13620, Korea Tel: +82-31-787-7194, Fax: +82-31-787-4056

E-mail: khkoo@snu.ac.kr
In 1971, Nishio and Sugioka ${ }^{11)}$ introduced TCVO (Fig. 1). In this procedure, a curved osteotomy is made between the greater and lesser trochanters. Then, the femoral head is rotated into a varus position. To date, five studies, four from Japan and one from Korea, have reported the results of TCVO. In these studies, the success rate ranged from $90 \%$ to $97.3 \%$. ${ }^{12-15)}$

In 1978, Sugioka" introduced another osteotomy, TRO (Fig. 2). In this technique, the greater trochanter is osteotomized, and the femoral head fragment is rotated anteriorly. The success rates of TRO were inconsistent ranging from $17 \%$ to $100 \%$. While studies from Japan and Korea reported successful results, ${ }^{16-19)}$ further collapse of the femoral head developed in $83 \%$ in a study from the United States. ${ }^{20)}$

The aim of this review is to provide up-to-date guides for the osteotomy of femoral head osteonecrosis.

\section{COMPARISON BETWEEN TCVO AND TRO}

There is no randomized clinical trial comparing TCVO and TRO. Thus far, only one study retrospectively com- 
Lee et al. Osteotomy for Osteonecrosis of the Femoral Head

Clinics in Orthopedic Surgery • Vol. 11, No. 2, 2019• www.ecios.org

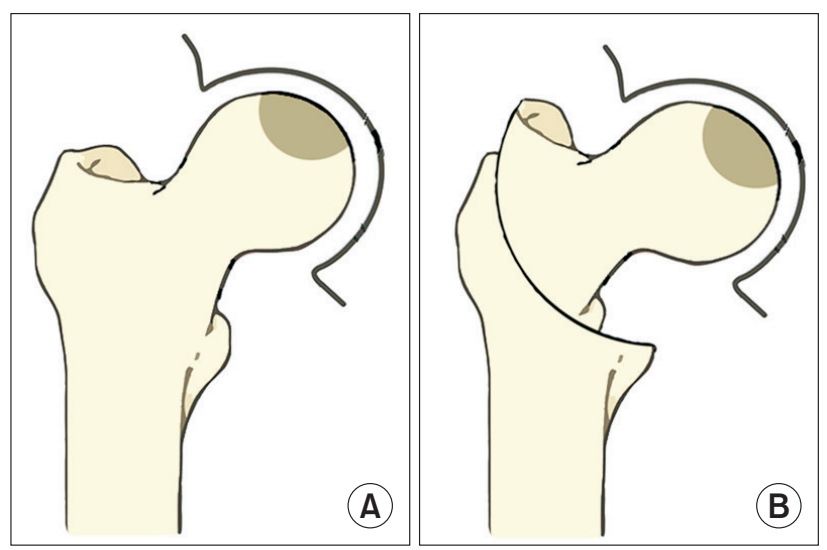

Fig. 1. Transtrochanteric curved varus osteotomy. (A) Osteonecrosis of femoral head. (B) A curved osteotomy is made between the greater and lesser trochanter, and the femoral head is rotated into a varus position.

pared these two osteotomies. Lee et al. ${ }^{15)}$ compared 85 patients (91 hips) who were treated with TRO and 58 patients (65 hips) who were treated with TCVO. The TCVO patients had shorter operation time and less blood loss. Postoperative collapse developed in 26 TRO hips (28.6\%) and seven TCVO hips (10.8\%). Osteophyte developed in 34 TRO hips (37.4\%) and 13 TCVO hips (20\%). Fifteen TRO hips (16.5\%) and seven TCVO hips (10.8\%) underwent conversion THA. The survival rate at 9 years with an endpoint of radiographic collapse was $68.7 \%$ in the TRO group and $84.7 \%$ in the TCVO group. With conversion to THA as the endpoint, the survival rate was $82.2 \%$ in the TRO group and $89.2 \%$ in the TCVO group. Their comparison showed that TCVO was better than TRO in terms of operation time, the amount of blood loss, postoperative collapse, osteoarthritic change and postoperative survival.

There are several differences between the two osteotomies. In TRO, the greater trochanter should be osteotomized and the joint capsule should be circumferentially incised. Accordingly, TRO necessitates longer operation time and more bleeding. In TCVO, the head segment is simply rotated into varus by about $30^{\circ}$ in the coronal plane. However, in TRO, the femoral head is rotated anteriorly by $60^{\circ}$ to $90^{\circ}$ and varization is required.

The femoral head is not perfectly spherical but elliptical. The greater axis lies horizontally; that is, the radius of curvature along the meridian is greater than the radius of curvature along the equator. In the study of Hammond and Charnley, ${ }^{21)}$ the mean difference of the two radiuses was $1.7 \mathrm{~mm}$. Therefore, the femoral head might become less congruous to the acetabulum after TRO than after TCVO. The mechanical simplicity and avoidance of cap-

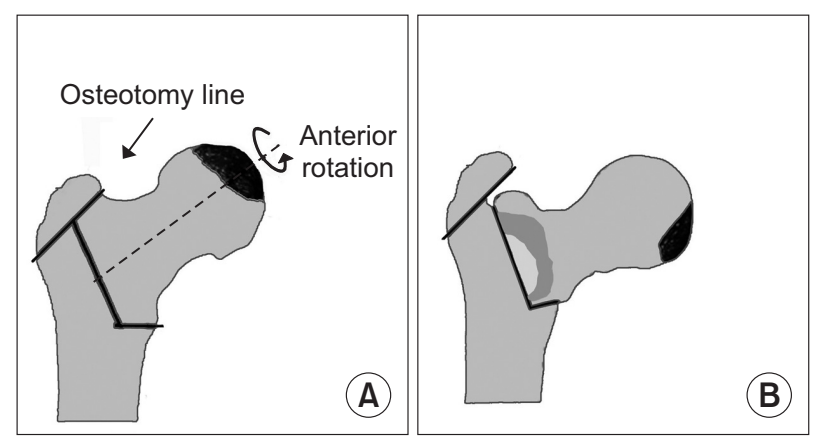

Fig. 2. Transtrochanteric rotational osteotomy. (A) The greater trochanter is osteotomized. (B) The femoral head fragment is rotated anteriorly.

sulotomy in TCVO seemed to result in less osteophyte formation compared to TRO.

\section{INDICATION OF THE OSTEOTOMY}

The reported results after the osteotomies were inconsistent. ${ }^{12-14,16-18,20)}$ Inappropriate patient selection is a reason for poor outcomes after the osteotomy; ${ }^{16,20)}$ to improve the success rate, more efficient selection of patients is mandatory. Patient's age, body mass index, the preoperative stage of the disease, the size of the necrotic portion, and remaining viable portion of the femoral head are known factors affecting the result after the osteotomy.

\section{Patient's Age and Body Mass Index}

Patient's age and body mass index are factors that affect results after the osteotomy. In a previous study, ${ }^{22)}$ secondary collapse was more frequent in patients who were aged $>40$ years and whose body mass index was $>24 \mathrm{~kg} / \mathrm{m}^{2}$. After the osteotomy, an intact bone is established in the weightbearing region of the femoral head. Secondary collapse is a stress fracture of this newly-established intact portion, which is usually thin and beak-shaped. Age-related osteopenia develops by the age of 40 years and progresses afterwards. ${ }^{23)}$ In patients with high body mass index, an excessive load is applied on the femoral head, which leads to a stress fracture and secondary collapse of the newly formed weight-bearing portion.

\section{Stage of the Disease}

Osteotomies should be performed in the early stages of the disease before marked collapse of the femoral head: Ficat stage IIB (a crescentic subchondral fracture or slight flattening of the femoral head) or stage III (a definite head collapse without joint space narrowing). ${ }^{24,25)}$ 


\section{Size of Necrotic Portion}

Small lesions do not progress even without any medical or surgical intervention; ${ }^{26)}$ whereas hips with a large lesion preoperatively have subsequent collapse of the femoral head after the osteotomy. ${ }^{27}$ Thus, the osteotomies should be performed in medium-size lesions with a combined necrotic angle between $190^{\circ}$ and $240^{\circ}$ (Fig. 3), ${ }^{28)}$ or type $\mathrm{B}$ lesions involving the medial two-thirds or less of the weight-bearing portion according to Japanese Investigation Committee (JIC) classification (Fig. 4). ${ }^{29)}$ The extent of necrotic portion should be measured on magnetic resonance imaging (MRI) for the accurate measurement of the necrotic portion.

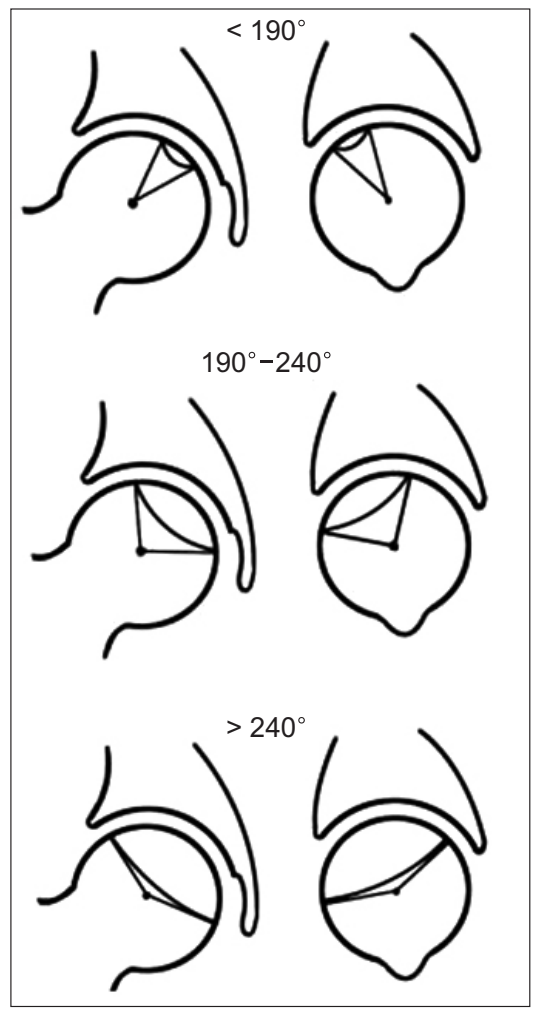

Fig. 3. The osteotomies should be performed in medium-size lesions with a combined necrotic angle between $190^{\circ}$ and $240^{\circ}$

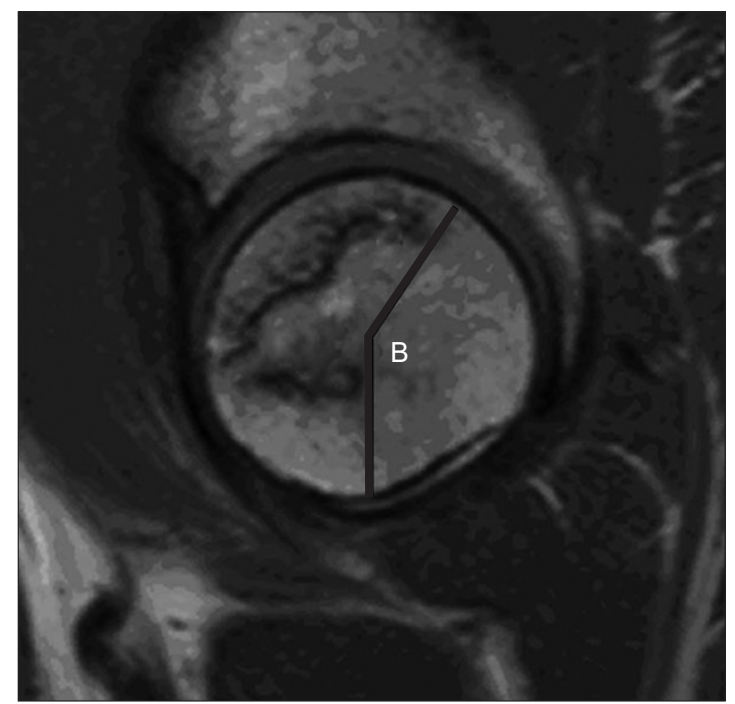

Fig. 5. Adequate area of viable bone for transtrochanteric rotational osteotomy is an arc (B) of $>120^{\circ}$ between the central vertical line of the femoral head and the posterior margin of the necrotic portion on a midsagittal magnetic resonance imaging scan.

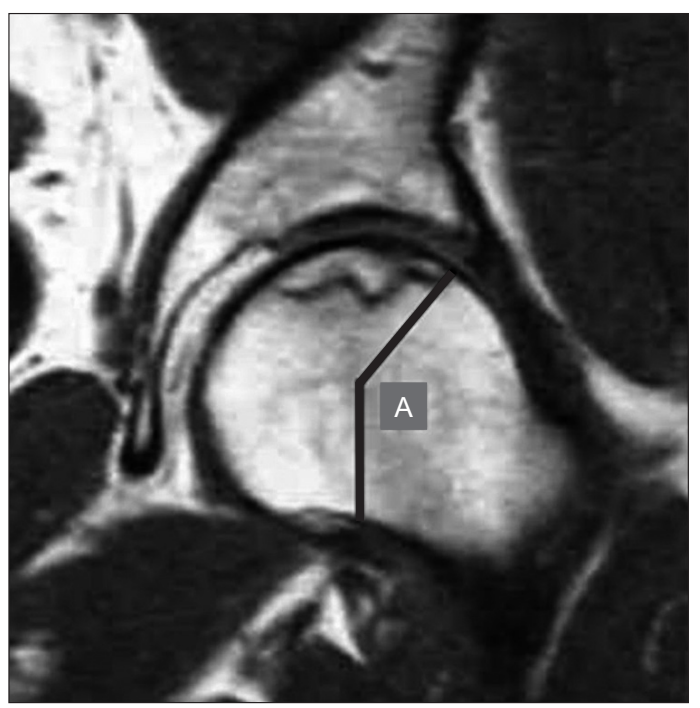

Fig. 6. Adequate area of viable bone for transtrochanteric curved varus osteotomy is an $\operatorname{arc}(A)$ of $>150^{\circ}$ between the central vertical line of the femoral head and the lateral margin of the necrotic portion on the midcoronal magnetic resonance imaging scan.

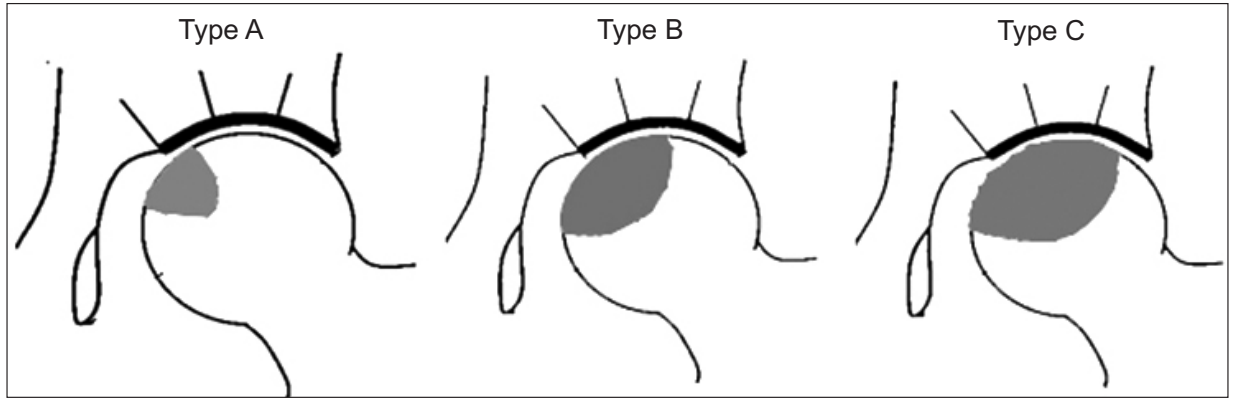

Fig. 4. The osteotomies should be performed in type $B$ lesions involving the medial two-thirds or less of the weightbearing portion according to Japanese Investigation Committee classification. 
Lee et al. Osteotomy for Osteonecrosis of the Femoral Head

Clinics in Orthopedic Surgery • Vol. 11, No. 2, 2019• www.ecios.org

\section{Viable Portion of the Femoral Head}

The femoral head should have a viable portion of such a size that restoration of an adequate weight-bearing articular surface is possible after the osteotomy. ${ }^{30,31)}$ The adequate area of viable bone for TRO is an $\operatorname{arc}$ of $>120^{\circ}$ between the central vertical line of the femoral head and the posterior margin of the necrotic portion on a midsagittal MRI scan (Fig. 5), and that for TCVO is an arc of $>150^{\circ}$ between the central vertical line of the femoral head and the lateral margin of the necrotic portion on a midcoronal MRI scan (Fig. 6).

\section{CONCLUSIONS}

We recommend the use of TCVO for the treatment of femoral head osteonecrosis in patients who have (1) hip pain, (2) age less than 40 years, (3) a body mass index less than $24 \mathrm{~kg} / \mathrm{m}^{2}$, (4) the Ficat stage IIA or III disease, (5) a medium-size lesion (combined necrotic angle between $190^{\circ}$ and $240^{\circ}$ or JIC type B lesion), and (6) enough viable bone $\left(>150^{\circ}\right.$ between the central vertical line and the lateral margin of the necrotic portion on the midcoronal MRI).

\section{CONFLICT OF INTEREST}

No potential conflict of interest relevant to this article was reported.

\section{REFERENCES}

1. Gutierrez F, Padilla S, Masia M, et al. Osteonecrosis in patients infected with HIV: clinical epidemiology and natural history in a large case series from Spain. J Acquir Immune Defic Syndr. 2006;42(3):286-92.

2. Shimizu K, Moriya H, Akita T, Sakamoto M, Suguro T. Prediction of collapse with magnetic resonance imaging of avascular necrosis of the femoral head. J Bone Joint Surg Am. 1994;76(2):215-23.

3. Shibatani M, Fujioka M, Arai Y, et al. Degree of corticosteroid treatment within the first 2 months of renal transplantation has a strong influence on the incidence of osteonecrosis of the femoral head. Acta Orthop. 2008;79(5):631-6.

4. Shigemura T, Nakamura J, Kishida S, et al. Incidence of osteonecrosis associated with corticosteroid therapy among different underlying diseases: prospective MRI study. Rheumatology (Oxford). 2011;50(11):2023-8.

5. Arlet J. Nontraumatic avascular necrosis of the femoral head: past, present, and future. Clin Orthop Relat Res. 1992;(277):12-21.

6. Baek SH, Kim SY. Cementless total hip arthroplasty with alumina bearings in patients younger than fifty with femoral head osteonecrosis. J Bone Joint Surg Am. 2008;90(6):131420.

7. Kim YH, Choi Y, Kim JS. Cementless total hip arthroplasty with ceramic-on-ceramic bearing in patients younger than 45 years with femoral-head osteonecrosis. Int Orthop. 2010;34(8):1123-7.

8. Fye MA, Huo MH, Zatorski LE, Keggi KJ. Total hip arthroplasty performed without cement in patients with femoral head osteonecrosis who are less than 50 years old. J Arthro- plasty. 1998;13(8):876-81.

9. Sugioka Y. Transtrochanteric anterior rotational osteotomy of the femoral head in the treatment of osteonecrosis affecting the hip: a new osteotomy operation. Clin Orthop Relat Res. 1978;(130):191-201.

10. Atsumi T, Muraki M, Yoshihara S, Kajihara T. Posterior rotational osteotomy for the treatment of femoral head osteonecrosis. Arch Orthop Trauma Surg. 1999;119(7-8):388-93.

11. Nishio A, Sugioka Y. A new technique of the varus osteotomy at the upper end of the femur. Orthop Traumatol. 1971;20(3):381-6.

12. Sakano S, Hasegawa Y, Torii Y, Kawasaki M, Ishiguro N. Curved intertrochanteric varus osteotomy for osteonecrosis of the femoral head. J Bone Joint Surg Br. 2004;86(3):35965.

13. Ikemura S, Yamamoto T, Jingushi S, Nakashima Y, Mawatari T, Iwamoto Y. Leg-length discrepancy after transtrochanteric curved varus osteotomy for osteonecrosis of the femoral head. J Bone Joint Surg Br. 2007;89(6):725-9.

14. Zhao G, Yamamoto T, Ikemura S, et al. Radiological outcome analysis of transtrochanteric curved varus osteotomy for osteonecrosis of the femoral head at a mean follow-up of 12.4 years. J Bone Joint Surg Br. 2010;92(6):781-6.

15. Lee YK, Park CH, Ha YC, Kim DY, Lyu SH, Koo KH. Comparison of surgical parameters and results between curved varus osteotomy and rotational osteotomy for osteonecrosis of the femoral head. Clin Orthop Surg. 2017;9(2):160-8.

16. Inao S, Ando M, Gotoh E, Matsuno T. Minimum 10-year results of Sugioka's osteotomy for femoral head osteonecrosis. Clin Orthop Relat Res. 1999;(368):141-8. 
Lee et al. Osteotomy for Osteonecrosis of the Femoral Head

Clinics in Orthopedic Surgery • Vol. 11, No. 2, $2019 \bullet$ www.ecios.org

17. Koo KH, Song HR, Yang JW, Yang P, Kim JR, Kim YM. Trochanteric rotational osteotomy for osteonecrosis of the femoral head. J Bone Joint Surg Br. 2001;83(1):83-9.

18. Tooke SM, Amstutz HC, Hedley AK. Results of transtrochanteric rotational osteotomy for femoral head osteonecrosis. Clin Orthop Relat Res. 1987;(224):150-7.

19. Ha YC, Kim HJ, Kim SY, Kim KC, Lee YK, Koo KH. Effects of age and body mass index on the results of transtrochanteric rotational osteotomy for femoral head osteonecrosis. J Bone Joint Surg Am. 2010;92(2):314-21.

20. Dean MT, Cabanela ME. Transtrochanteric anterior rotational osteotomy for avascular necrosis of the femoral head: long-term results. J Bone Joint Surg Br. 1993;75(4):597-601.

21. Hammond BT, Charnley J. The sphericity of the femoral head. Med Biol Eng. 1967;5(5):445-53.

22. Ha YC, Kim HJ, Kim SY, Kim KC, Lee YK, Koo KH. Effects of age and body mass index on the results of transtrochanteric rotational osteotomy for femoral head osteonecrosis: surgical technique. J Bone Joint Surg Am. 2011;93 Suppl 1:75-84.

23. Parfitt AM. Age-related structural changes in trabecular and cortical bone: cellular mechanisms and biomechanical consequences. Calcif Tissue Int. 1984;36 Suppl 1:S123-8.

24. Sugano N, Takaoka K, Ohzono K, Matsui M, Saito M, Saito S. Rotational osteotomy for non-traumatic avascular necrosis of the femoral head. J Bone Joint Surg Br. 1992;74(5):734-9.

25. Hisatome T, Yasunaga Y, Takahashi K, Ochi M. Progressive collapse of transposed necrotic area after transtrochanteric rotational osteotomy for osteonecrosis of the femoral head induces osteoarthritic change: mid-term results of transtrochanteric rotational osteotomy for osteonecrosis of the femoral head. Arch Orthop Trauma Surg. 2004;124(2):7781.

26. Mont MA, Zywiel MG, Marker DR, McGrath MS, Delanois RE. The natural history of untreated asymptomatic osteonecrosis of the femoral head: a systematic literature review. J Bone Joint Surg Am. 2010;92(12):2165-70.

27. Mont MA, Fairbank AC, Krackow KA, Hungerford DS. Corrective osteotomy for osteonecrosis of the femoral head. J Bone Joint Surg Am. 1996;78(7):1032-8.

28. Ha YC, Jung WH, Kim JR, Seong NH, Kim SY, Koo KH. Prediction of collapse in femoral head osteonecrosis: a modified Kerboul method with use of magnetic resonance images. J Bone Joint Surg Am. 2006;88 Suppl 3:35-40.

29. Sugano N, Atsumi T, Ohzono K, Kubo T, Hotokebuchi T, Takaoka K. The 2001 revised criteria for diagnosis, classification, and staging of idiopathic osteonecrosis of the femoral head. J Orthop Sci. 2002;7(5):601-5.

30. Atsumi T, Kuroki Y. Modified Sugioka's osteotomy: more than 130 degrees posterior rotation for osteonecrosis of the femoral head with large lesion. Clin Orthop Relat Res. 1997;(334):98-107.

31. Hiranuma Y, Atsumi T, Kajiwara T, Tamaoki S, Asakura Y. Evaluation of instability after transtrochanteric anterior rotational osteotomy for nontraumatic osteonecrosis of the femoral head. J Orthop Sci. 2009;14(5):535-42. 